the apex beat was diffused. There were no increase in the superficial cardiac dulness and no bruits. The liver dulness commenced at the upper border of the sixth rib in the nipple line. On inspection the abdomen was found to be deeply jaundiced. Distension was noticed to the right of the umbilicus. On palpation to the right of, and extending for two or three inches below the level of, the umbilicus was a tender, elastic, tense resistance, roughly triangular in shape, merging above and on either side of its base with a more solid resistance, presumably the liver. On percussion the above regions were both dull. The physical signs thus pointing to some obstruction of the common bile duct exploratory laparotomy was advised.

On Dec. 6th Dr. Hatton made an incision in the linea semilunaris on the right side over the swelling and on opening the peritoneal cavity he discovered a much distended gall-bladder, bearing signs of an acute local inflammatory condition and adherent above to a portion of great omentum. Cellular blanket cloth swabs having been packed around the lower and outer aspects of the gall-bladder, an incision was made into it and a quantity of bile, at first purulent but later almost normal in character, was evacuated. The cavity of the gall-bladder was then found to be full of smooth small faceted calculi, averaging about the size of a large pea, of which 449 were removed. The gall-bladder was then sutured to the abdominal parietes and a drainage-tube and iodoform gauze-plug were inserted; the rpper portion of the wound was closed with silkworm-gut sutures. Owing to the length of the operation it was not deemed advisable to explore the gall-ducts at this time.

The patient bore the operation well and was anæsthetised with ether. The after progress was very satisfactory ; the jaundice lessened and disappeared. The tube was removed on Dec. 8th and the stitches on the 16th. 'Two more calculi of small size were passed, one on the 12 th and the other on the 27th, bringing the total up to 451 . The biliary fistula slowly closed, normal bile being passed but lessening gradually and on the patient's leaving the infirmary on Jan. 12th, 1903, all that remained was a button of granulations with slight exudation of bile.

Stoke-on-Trent

\section{A CASE OF COMPOUND COMMINUTED FRACTURE OF THE SKULL WITH HERNIA CEREBRI; OPERATION ; RECOVERY.}

BY LLEWELYN LEWIS, M.R.C.S. ENG., L.R.C.P. LOND

A Boy, aged 11 years, was seen at his home at 11.30 A.M. on April 3rd, 1902. The mother stated that he had been kicked by a horse one and a half hours previously and that he had lost a tremendous quantity of blood. Previously the boy had been healthy and strong. The father of the boy was a miner living in a small cottage of two rooms. The family history was good. When first seen the boy was lying on a settle. His face was blanched and blood was running from the right side of the head above the ear $\mathrm{He}$ was in a collapsed condition and unconscious. The pulse was very small.

On examination a lacerated wound was found, one inch in length, situated two and a half inches from the root of the zygoma, four inches from the middle of the bregma, four and a half inches from the middle line of the forehead, three and a half inches from the upper edge of the mastoid process, running horizontally, the centre of the wound being in a line one-eighth of an inch in front of the external auditory meatus. Brain substance was oozing out. Palpation revealed a compound fracture of the skull, the lower part of the bone being driven in and the upper edge of the bone projecting.

The hair was shaved around the wound and the scalp was thoroughly cleansed with a 1 in 2000 solution of biniode of mercury and dressed with antiseptic wool. His parents were advised to remove him to the hospital and the seriousness of the case was pointed out to them. Up to this time the history is as given to me by Dr. W. Evans, who was then assisting me in my practice. On the following morning a message was received by me asking that something migbt be done for the boy, as the mother would not allow him to be taken from home. At 1 P.M. (27 hours after the accident) the boy was seen and he was then in the same position and condition as on the previous day.
At 5 P. M. (31 hours after the accident) Dr. Evans and I saw him and there was no difficulty in concluding that the case was one of compound fracture of the skull with protrusion of brain matter. The boy was at this time in bed in the one living room of the house. He had to some extent recovered consciousness but was very dazed and looked ill. He was at once put under an anxsthetic and the right half of the scalp was shaved and made as aseptic as possible. A semicircular incision was made through the scalp covering the area of injury. On reflecting this flap more brain matter was seen to be oozing out of the cranial cavity and it was seen that the fracture was comminuted and that the posterior half was forced down and its edge deeply driven into the brain. The anterior part of the fractured portion was also broken up into several pieces, some being forced into the brain. Being unable to remove the fragments of bone a trephine was applied to the sound portion of the skull on the upper edge of the fracture and an almost complete circle of bone was removed. A lever was then inserted under the depressed fragment, but as this did not enable me to remove it, a strong polypus forceps was used and the bone at the posterior of the fracture was wrenched out and raised above the level of the skull. In endeavouring to replace this it was found that the portion was now too broad to fit into its original place, by the loosening of the suture between the parietal and squamous portion of the temporal bone; the fragment was therefore entirely removed and this, with the other fragments, was placed in a normal saline solution at a temperature of about $100^{\circ} \mathrm{F}$. On further examination of the parts portions of brain matter were discovered to be so injured that it was deemed necessary to remove them and this was done by the aid of scissors. The brain and surrounding parts were then thoroughly cleansed with a 1 in 60 solution of phenic acid, the dura mater was brought together and the pieces of the skull were then sawn into smaller ones and filled into the space like tessellated pavement. A drainage-tube was passed down to the fracture, the scalp was brought together with fishing-gut sutures, and the whole was dressed with perchloride wool.

By the time we had washed ourselves and the instruments the boy had recovered consciousness and from that hour his intellect has been perfectly clear. His temperature did not once rise above normal and with the exception of a little swelling over the left eye, the recovery was uninterrupted. The drainage-tube was removed on the fourth day and the whole wound quickly healed by first intention. The skull is now solid and all the pieces of bone have united to each other. There was on no occasion any symptom of paresis in any organ or muscle and the pupils remained normal throughout and responded to light and accommodation. His hearing was not in any way impaired. There has not been any symptom of brain trouble since and the boy is as formerly.

The following three points are worthy of consideration: (1) the recovery in a most insanitary environment, the room in which the lad was lying being the only living room of a family of nine, the work of the household being carried on all the time ; (2) the absence of any symptom of impairment, either mentally or physically; and (3) the exceilent union of the sawn particles of bone which were immersed in saline solution for a period of not less than one and a balf hours. From measurements taken it is assumed that the region injured was part of the temporo-sphenoidal lobe. Neath.

London Tuberculosis Sanatoriums. - At a meeting of the Metropolitan Asylums Board on Feb. 7th letters were read from the Shoreditch, St. Marylebone, Paddington, and Stoke Newington borough councils stating that they agreed with the suggestion of the Kensington borough council that the Orchard and Joyce Green smallpox hospitals should be set apart for the treatment of tuberculous persons. Some months ago the Bermondsey borough council, after circularising the other councils of London on the same subject, found that ten were in favour of sanatoriums being provided by the Metropolitan Asylums Board ; five approved of the principle of the need for the open-air treatment of buberculous patients but were not agreed as to the authority which should make the provision and 11 remained indifferent. On that occasion the Board decided to wait until the borough councils came into line and a year was allowed for that purpose. At the meeting on Feb. 7th, however, the letters gave rise to some discussion and notice of motion was given that the whole subject be reconsidered at the next meeting of the Board. 